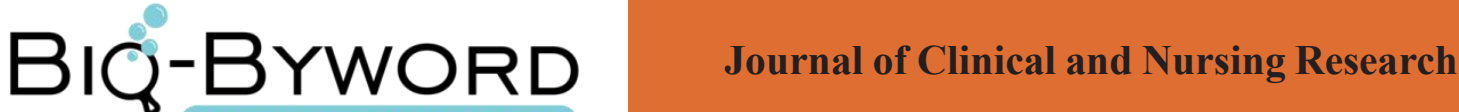

\section{Application of Nursing Safety Management in Orthopedic Nursing Management}

Han Fang

New Hospital of Xinyi City, Jiangsu Province, Xinyi 221400

\section{ARTICLE INFO}

Article history:

Published online: $30^{\text {th }}$ Sept, 2017

\section{Key words:}

Nursing safety management

Orthopedic care

Nursing level

Nursing satisfaction

\begin{abstract}
Objective: To analyze the application of nursing safety management in orthopedic nursing management. Methods: From January 2015 to December 2015, the Department of Orthopedic Nursing Safety management measures, as well as January 2016 December 2016 during the period of our hospital for orthopedic care safety management measures, the nursing work to sort out the information Statistics, and the two measures before and after the implementation of our hospital orthopedic care level and patient care satisfaction were retrospectively summed up, and the value of the comparison. Results: After the implementation of orthopedic nursing safety management measures, the nursing level of our hospital was obviously improved, and the patient 's nursing satisfaction increased significantly, the difference was statistically significant $(\mathrm{P}<0.05)$. Conclusion: In view of the existing problems and safety risks in the orthopedic nursing work in our hospital, the nursing management measures were implemented, and the management system of orthopedic care was improved to improve the level of orthopedic care and improve the patient's nursing satisfaction. Patients with injuries caused by the safety of hidden dangers, worthy of our hospital in the future orthopedic care safety management system to improve the work of the promotion and application.
\end{abstract}

\section{Introduction}

With the development of society, the living standard gradually increased, the medical level is also developing rapidly. People in medical treatment, not only for the treatment of the effect began to pay attention to the treatment process for nursing staff care and nursing safety are more to the higher requirements, and recent news reports, the data show that the increase in medical disputes, which makes the medical sector for nursing management measures are attracting more and more attention. How to effectively deal with the safety of care hidden dangers, and improve the level of care and care satisfaction, is also more important research projects.

\subsection{General information}

In the period from January 2015 to December 2015, there will be a retrospective review of the nursing work before the orthopedic care safety management measures in our hospital and during the period from January 2016 to December 2016. A total of 276 patients were treated with orthopedic treatment. The patients were between 10 and 85 years old and had a body weight of 46.2 to $75.1 \mathrm{~kg}$ with an average $(59.2 \pm 2.1) \mathrm{kg}$. A total of 281 patients underwent orthopedic treatment. The patients were between 12 and 84 years old and had a body weight of $48.1-87.1 \mathrm{~kg}$ with an average $(48.4 \pm 2.6) \mathrm{kg}$. There were no significant differences in the general data between the two orthopedic patients $(\mathrm{P}>0.05)$.

\section{Information and methods}




\subsection{Research methods}

The main measures include: 1) Nursing staff training level training: to the orthopedic nursing staff of professional medical knowledge and emergency treatment measures for training, especially in the course of orthopedic treatment, sudden treatment of orthopedic care. The situation is different, so it is necessary to strengthen the training of orthopedic care staff of patients with care ability, as well as patients with emergency treatment approach. 2) The establishment and improvement of the system: the most important part of nursing safety management is the nursing process and the establishment of management system, to the nursing staff shift system, medication management system and nursing process specifications, set a perfect and formal of the rules and regulations, requiring nurses to strictly follow the process of nursing work, in which to reduce care in the security risks. 3) The safety of the ward: the hospital staff is more complicated, so for orthopedic patients, especially patients with major surgery or seriously ill patients, must ensure safety and to avoid accidents.

\subsection{Evaluation indicators}

The degree of care of the orthopedic nursing staff before and after the implementation of the measures was carried out by

the senior nursing officer, and the scores of the nursing care satisfaction survey were summarized and counted.

\subsection{Data processing}

The data of this study were statistically analyzed by SPSS 17.0 software. The nursing level was measured by $(\mathrm{x} \pm \mathrm{s})$. The t-test and nursing satisfaction were used as the counting data in terms of rate (\%), test comparison. $\mathrm{P}<0.05$ that the difference was statistically significant.

\section{Results}

After the implementation of orthopedic care safety management measures, the level of nursing care in our hospital was significantly improved, and the patient's nursing satisfaction increased significantly, the difference was statistically significant $(\mathrm{P}<0.05)$. See Table 1 for details.

Table 1 Nursing safety management measures before and after the implementation of care levels, nursing satisfaction, etc. $(\mathrm{x} \pm \mathrm{s})$

\begin{tabular}{cccc}
\hline Time & Number & $\begin{array}{c}\text { Nursing Level } \\
\text { (Score) }\end{array}$ & $\begin{array}{c}\text { Nursing Satisfaction } \\
{[\mathrm{n}(\%)]}\end{array}$ \\
\hline Before implementation & $\mathrm{n}=276$ & $77.58 \pm 5.21$ & $201(73.00)$ \\
After implementation & $\mathrm{n}=281$ & $\begin{array}{c}89.64 \pm 4.56 \\
\mathrm{P}<0.05\end{array}$ & $\begin{array}{c}268(95.00) \\
\mathrm{P}<0.05\end{array}$ \\
\hline
\end{tabular}

Note: Compared with the control group, ${ }^{*} \mathrm{P}<0.05$

\section{Discussion}

With the patient's level of treatment requirements gradually increased, and the clinical medicine community slowly found the importance of care for the improvement of treatment, especially in recent years, orthopedic care has been low in satisfaction, and which contains more security risks, so in recent years, for the management of orthopedic care system measures are gradually improved. Nursing safety management is based on the patient as the center, for the nursing process of the existence of security risks and problems, the establishment of a sound nursing management measures, the care of the nursing staff and the patient's care satisfaction to improve, in which not only it is necessary to adjust the nursing ability and crisis management ability of nurses, and standardize the workflow of nursing staff, reduce the emergence of dangerous events in nursing, strengthen the trust of patients and reduce the occurrence of medical disputes.

\section{Conclusion}

In summary, for our hospital orthopedic nursing work in 